\title{
An incomplete set of shortest descriptions*
}

\author{
Frank Stephan \\ National University of Singapore \\ Department of Mathamtics \\ 10 Lower Kent Ridge Road, Singapore 119076 \\ fstephan@comp.nus.edu.sg \\ Jason Teutsch \\ Ruprecht-Karls-Universität Heidelberg \\ Institut für Informatik \\ Im Neuenheimer Feld 294, 69120 Heidelberg, Germany \\ teutsch@math.uni-heidelberg.de
}

October 27, 2011

\begin{abstract}
The truth-table degree of the set of shortest programs remains an outstanding problem in recursion theory. We examine two related sets, the set of shortest descriptions and the set of domain-random strings, and show that the truth-table degrees of these sets depend on the underlying acceptable numbering. We achieve some additional properties for the truth-table incomplete versions of these sets, namely retraceability and approximability. We give priority-free constructions of bounded truth-table chains and bounded truth-table antichains inside the truth-table complete degree by identifying an acceptable set of domain-random strings within each degree.
\end{abstract}

\section{Meyer's Problem}

No algorithm can determine, even in the limit, whether two distinct programs represent the same function. But one can, relative to the set of shortest programs

$$
\operatorname{MIN}_{\varphi}=\left\{e:(\forall j<e)\left[\varphi_{j} \neq \varphi_{e}\right]\right\}
$$

${ }^{*}$ This work was supported by NUS grant R252-000-420-112 (F. Stephan) and DFG grant ME 1806/3-1 (J. Teutsch). 
figure out in the limit whether $\varphi_{d}=\varphi_{e}$. Here $\varphi$ denotes any reasonable programming language or "acceptable numbering" in which one can effectively code any algorithm. Members of $\mathrm{MIN}_{\varphi}$ are not only the most concise programs in the language $\varphi$; they must in fact be minimal.

How difficult is it to decide whether a piece of code belongs to the set of shortest programs? Meyer posed this question in a 1972 paper [20]. He showed that $\operatorname{MIN}_{\varphi}$ is Turing-equivalent to $K^{\prime}$ for every acceptable numbering $\varphi$ but left the truth-table degree of this set as an open problem.

Question 1.1. Is $\operatorname{MIN}_{\varphi} \equiv_{\mathrm{tt}} K^{\prime}$ for every acceptable numbering $\varphi$ ?

The truth-table degree of $\operatorname{MIN}_{\varphi}$ remains a mystery even today.

Five years after Meyer introduced his problem, Kinber [13] constructed acceptable numberings $\varphi$ and $\psi$ such that $\operatorname{MIN}_{\varphi}$ and $\operatorname{MIN}_{\psi}$ have distinct bounded truth-table degrees. In a follow-on paper, Marandžjan showed that the conjunctive degree of $\mathrm{MIN}_{\varphi}$ can vary for acceptable numberings as well [18], [19]. Kinber further mentions the existence of an acceptable numbering $\varphi$ satisfying $\operatorname{MIN}_{\varphi} \equiv_{\mathrm{tt}} K^{\prime}$. Two decades after Kinber's seminal work, Schaefer upgraded $\varphi$ to a Kolmogorov numbering satisfying $\operatorname{MIN}_{\varphi} \equiv_{\text {tt }} K^{\prime}$ [25]. Schaefer also points out that $\operatorname{MIN}_{\varphi} \geq_{\text {wtt }} K$ for all acceptable numberings $\varphi$ but leaves the weak truth-table degree of $\operatorname{MIN}_{\varphi}$ as an important related problem.

This exposition draws on concepts from both recursion theory and algorithmic randomness. Given the intimate relationship between randomness and incompressibility, it is no surprise that minimal indices, as highly compressed objects themselves, also associate closely with randomness. A numbering $\varphi$ is a partial-recursive function $\langle e, x\rangle \mapsto \varphi_{e}(x)$; a numbering $\varphi$ is called a (K-) acceptable numbering if for every further numbering $\psi$ there exists a (K-)recursive function $f$ such that $\varphi_{f(e)}=\psi_{e}$ for all $e$. Here $K$ denotes the halting set. If $\varphi$ is acceptable and in addition for every $\psi$ the corresponding translation function $f$ is linearly bounded, then $\varphi$ is a Kolmogorov numbering. If $f$ is just linear (and not necessarily recursive) we say that $f$ has the Kolmogorov property. Having a numbering $\varphi$ with the Kolmogorov property, one can also define the plain Kolmogorov complexity $C$ of a number $x$ as $C(x)=\min \left\{\log (e): \varphi_{e}(0)=x\right\}$ where this value depends only up to a constant on the underlying numbering.

A left-r.e. set $X$ is a set which has a uniformly recursive approximation $X_{0}, X_{1}, \ldots$ such that $X_{s} \leq_{\text {lex }} X_{s+1}$ for all $s$. Here $X_{s} \leq_{\operatorname{lex}} X_{s+1}$ iff either $X_{s}=X_{s+1}$ or the least element of the symmetric difference is in $X_{s+1}$. There is some correspondence between left-r.e. sets and real numbers which are approximable from below: for every set $X$ there is the real number 
$r_{X}=\sum_{n} 2^{-n-1} X(n)$ where $r_{X}=r_{Y}$ can only happen for distinct $X, Y$ when $X, Y$ are finite and cofinite, respectively. Zvonkin and Levin [33] and later Chaitin [4], [5] constructed a left-r.e. Martin-Löf random set [17] which we call $\Omega$. Every Martin-Löf random set $X$ satisfies $C(X \| n)>n / 2$ for all but finitely many $n$ [7]. For the purposes of this paper, the property of $\Omega$ described in the preceding sentence suffices; the actual characterization of Martin-Löf random is much stronger but also a bit more complicated to formulate (as one either needs prefix-free Kolmogorov complexity or has to deal with the fact that the plain complexity is only almost above $n$ but not exactly above $n$ ).

Definition 1.2. A set $A$ is truth-table reducible to $B$, written $A \leq_{\mathrm{tt}} B$, if there exist recursive functions $f$ and $g$ such that for all $x$,

$$
x \in A \quad \Longleftrightarrow \quad f[x, B(0), B(1), \ldots, B(g(x))]=1 .
$$

Furthermore, $A$ is truth-table complete if $A \equiv_{\mathrm{tt}} K$. Intuitively, $A \leq_{\mathrm{tt}} B$ if membership in $A$ can be decided by constructing a Boolean formula and evaluating it using membership values from $B$. If the truth-table $f$ can be chosen so as to depend on only a constant number of membership queries from $B$, then a stronger reduction is satisfied, namely $A \leq_{\mathrm{btt}} B$. We say $A \leq_{\text {wtt }} B$ if (1.1) is achieved for correct values $B(0), B(1), \ldots$ but $f$ is not necessarily total. A set $A$ is btt-complete if $A \equiv_{\mathrm{btt}} K$ and similarly for $\leq_{\mathrm{wtt}}$.

A set is called $n$-r.e. if it has a recursive approximation (starting from the empty set) with at most $n$ mind-changes on any given index. Here "r.e." stands for recursively enumerable. A set is $\omega$-r.e. if it has a recursive approximation with a recursive bound on the number of mind-changes for each index. A co-n-r.e. set is the complement of an $n$-r.e. set. A set $A$ satisfies $A \leq_{\text {btt }} K$ iff $A$ is $n$-r.e. for some $n$, and $A \leq_{\text {tt }} K$ iff $A$ is $\omega$-r.e. [11]. A collection of sets is called a (btt)-antichain if any two members of the collection are incomparable under (btt)-reductions. A collection of sets $A_{0}, A_{1}, \ldots$ satisfying $A_{0}<_{\mathrm{btt}} A_{1}<_{\mathrm{btt}} \ldots$ is a btt-ascending chain and if $A_{0}>_{\text {btt }} A_{1}>_{\text {btt }} \ldots$ then $A_{0}, A_{1}, \ldots$ is a descending chain.

An infinite set is called immune if it contains no infinite r.e. subset. A set which is immune and has a complement which is immune is bi-immune. Let $D_{0}, D_{1}, \ldots$ be a canonical enumeration of the finite sets. A set $A$ is $\omega$ immune if there is no recursive function $f$ and number $k$ such that $\left\{D_{f(i)}\right\}$ is a pairwise disjoint collection of sets, each with cardinality $k$, where each set has non-trivial intersection with $A$. If instead of $k$ the cardinality is a recursive function of the index, then $A$ is hyperimmune. 
We use ' to denote the jump operator, $\equiv_{\mathrm{T}}$ to denote Turing equivalence, $\equiv_{\mathrm{m}}$ to mean many-one equivalence and $=^{*}$ to mean equal except for a finite set. The symbols $\psi, \varphi$ refer to numberings and $W_{e}^{\varphi}$ is the domain of $\varphi_{e}$. $A \| n$ denotes $A(0) A(1) \ldots A(n)$. The symbol $\downarrow$ means converge, and $\uparrow$ means diverge. The letter $K$ denotes the halting set and is used in the full form $\left\{\langle e, x\rangle: x \in W_{e}\right\}$ so that the choice of the numbering does not influence the many-one degree. For further background on minimal indices, see Schaefer's survey article [25] or the more recent papers [30], [31]. For background and notation on recursion theory, see [21] and [27].

\section{The set of shortest descriptions}

In a formal sense, the set of shortest descriptions,

$$
\mathrm{SD}_{\varphi}=\left\{e:(\forall j<e)\left[\varphi_{j}(0) \neq \varphi_{e}(0)\right]\right\},
$$

is a simpler object than the set of shortest programs. Roughly speaking the former deals with enumerations of integers whereas the latter pertains to enumerations of functions. Schaefer invented the set of shortest descriptions for his Master's Thesis at University of Chicago [25]. There he proved that $\mathrm{SD}_{\varphi} \equiv_{\text {wtt }} K$ for every acceptable numbering $\varphi$. Together with Fenner, Schaefer further proved that $\mathrm{SD}_{\varphi} \searrow_{\text {btt }} K$ for every acceptable numbering $\varphi$ [8], [25]. Turning to the natural intermediate reduction between $\leq_{\text {btt }}$ and $\leq_{\text {wtt }}$, Schaefer [25] then asked whether $\mathrm{SD}_{\varphi}$ has to be truth-table complete for all acceptable numberings $\varphi$. The answer is "no" and now an acceptable numbering is constructed where $\mathrm{SD}_{\varphi}$ has the same truth-table degree as Chaitin's $\Omega$, a set known to be truth-table incomplete [2]. We note that there is a further Kolmogorov numbering $\psi$ for which $\mathrm{SD}_{\psi}$ is truth-table complete [31], [32].

Definition 2.1. A set $A=\left\{a_{0}<a_{1}<a_{2} \ldots\right\}$ is retraceable if there exists a partial-recursive function $f$ satisfying $f\left(a_{n+1}\right) \downarrow=a_{n}$ for all $n$.

Definition 2.2. A set $S$ is approximable if there exists an $n$ and a recursive function $f$ such that for any $x_{1}<\ldots<x_{n}$, the $n$-bit vector $f\left(x_{1}, \ldots, x_{n}\right)$ agrees with the characteristic vector $S\left(x_{1}, \ldots, x_{n}\right)$ in at least one place. In particular, we say $S$ is $(1, n)$-recursive.

The notions of "retraceable" and "approximable" may be thought of as generalizations of the notion of "recursive". For background on on retraceable sets, see Odifreddi's book [21]. For approximable sets, see [16]. We view the following theorem as progress towards Meyer's problem. 
Theorem 2.3. There exists an acceptable numbering $\psi$ such that:

(I) $\mathrm{SD}_{\psi} \Varangle_{\mathrm{tt}} K$,

(II) $\mathrm{SD}_{\psi}$ is retraceable and approximable.

Proof. (I). It is known that Chaitin's $\Omega$ satisfies $K \not \mathbb{t t}_{\mathrm{tt}} \Omega$ [2]. Therefore it suffices to find an acceptable numbering $\psi$ such that $\mathrm{SD}_{\psi} \leq_{\mathrm{tt}} \Omega$. In fact we shall build $\psi$ in such a way that $\mathrm{SD}_{\psi} \equiv_{\mathrm{tt}} \Omega$.

Let $\Omega_{0}, \Omega_{1}, \ldots$ be an uniformly recursive approximation of $\Omega$ from the left. Define for each $n$ the number

$$
a_{n}=2^{n} \Omega(0)+2^{n-1} \Omega(1)+\ldots+2^{1} \Omega(n-1)+2^{0} \Omega(n)+1 .
$$

This sequence satisfies $C\left(a_{n}\right)>n / 2$ for almost all $n$, as Chaitin's $\Omega$ is Martin-Löf random. Furthermore, let $I_{0}, I_{1}, I_{2}, \ldots$ be a splitting of the natural numbers into intervals of natural numbers such that each interval $I_{n}$ consists of $2^{n+1}$ subintervals, $J_{n, 2^{n+1}}, \ldots, J_{n, 2}, J_{n, 1}$ where $J_{n, k}$ has length $k$ and the ordering of the intervals is so that $\min \left(J_{n, k}\right)>\max \left(J_{n, k+1}\right)$ for the corresponding $k$. Furthermore, there is a subinterval $H_{n}$ of length 1 which sits immediately above all the $J_{n, k}$ and contains the maximal element $e_{n}$ of $I_{n}$. We can visualize the ordering on these intervals as follows:

$$
\underbrace{J_{0,2} J_{0,1} H_{0}}_{I_{0}} \underbrace{J_{1,4} J_{1,3} J_{1,2} J_{1,1} H_{1}}_{I_{1}} \underbrace{J_{2,8} J_{2,7} J_{2,6} J_{2,5} J_{2,4} J_{2,3} J_{2,2} J_{2,1} H_{2}}_{I_{2}} \cdots
$$

Note that this partitioning and the mapping $n \mapsto e_{n}$ are both recursive.

In order to make $\psi$ an acceptable numbering, we define $\psi_{e_{n}}(k)=\varphi_{n}(k)$ for all $k>0$. We would also like to define $\psi_{e_{n}}(0)=\varphi_{n}(0)$, but for technical reasons this property will only be satisfied at all but finitely many $n$. Second, one defines some $\psi$-indices $i \notin\left\{e_{0}, e_{1}, e_{2}, \ldots\right\}$ to take constant functions so as to achieve

$$
\mathrm{SD}_{\psi}={ }^{*} \bigcup_{n} J_{n, a_{n}} \equiv_{\mathrm{tt}} \Omega \text {. }
$$

Indeed, $\bigcup_{n} J_{n, a_{n}} \leq_{\mathrm{tt}} \Omega$ as $a_{n}$ can be determined from queries to $\Omega$ at its first $n+1$ values. On the other hand $\Omega(n)=1$ iff $a_{n}$ is even and $a_{n}$ can be determined by querying $\bigcup_{n} J_{n, a_{n}}$ on the interval $I_{n}$. So $\Omega \leq_{\mathrm{tt}} \bigcup_{n} J_{n, a_{n}}$.

The precise algorithm for indices lying in coding intervals $J_{n, k}$ is the following. At step 0, all functions in $J_{n, k}$ are undefined for all $n$ and $k$. In each step $t>0$, compute the values $a_{n, t}$ by using $\Omega_{t}$ in place of $\Omega$ :

$$
a_{n, t}=2^{n} \Omega_{t}(0)+2^{n-1} \Omega_{t}(1)+\ldots+2^{1} \Omega_{t}(n-1)+2^{0} \Omega_{t}(n)+1 .
$$


Now for $n=0,1,2, \ldots$, if $a_{n, t}=a_{n, t-1}$ then the functions in $J_{n, a_{n, t}}$ are already defined and nothing has to be done. Else do the following: for all $c$ such that either $\varphi_{n, t}(0)=c$ or such that the constant function $c^{\infty}(x)=c$ is one of the functions defined in $J_{n, a_{n, t-1}}$, check whether $c^{\infty}$ occurs already in some interval $J_{k, \ell}$ for some $k<n$ and $\ell \in\left\{1,2, \ldots, a_{k, t}\right\}$. If it does not, then take one index in $J_{n, a_{n, t}}$ and define this function to be $c^{\infty}$. Furthermore, fill the remaining indices in $J_{n, a_{n, t}}$ with new functions $c^{\infty}$ for values $c$ such that no $J_{k, \ell}$ with $k<n$ and $\ell \in\left\{1,2, \ldots, a_{k, t}\right\}$ contains an index for such a function.

Finally, for the remaining indices, define $\psi_{e_{n}}(0)=c$ iff $\varphi_{n}(0)=c$ and there is some index $i<e_{n}$ in a coding interval to which the constant function $c^{\infty}$ has been assigned. Otherwise $\psi_{e_{n}}(0)$ remains undefined. We now show that there are only finitely many $e_{n}$ for which $\psi_{e_{n}}(0)$ is undefined and $\varphi_{n}(0)$ is defined. Thus, up to finitely many errors, the mapping $n \mapsto e_{n}$ witnesses that $\varphi$ can be many-one reduced into the numbering $\psi$, hence the numbering $\psi$ is acceptable.

Note that whenever $\varphi_{n, t}(0)$ converges, the Kolmogorov complexities of $t$ and $a_{n, t}$ are both bounded by $\log (n)$ plus some constant. On the other hand, for almost all $n, C\left(a_{n}\right)>n / 2$ as $a_{n}$ codes the first $n$ bits of the Martin-Löf random $\Omega$. Hence, if $n$ is sufficiently large, then $a_{n, t}<a_{n}$ for all $t$ where $\varphi_{n, t}(0)$ is undefined but $\varphi_{n}(0)$ later converges. Furthermore whenever $a_{n, t+1}>a_{n, t}$, the corresponding interval $J_{n, a_{n, t+1}}$ will be larger than $J_{n, a_{n, t}}$, so a new index of the function $\varphi_{n}$ can be accommodated in stage $t+1$ while the old indices from $J_{n, a_{n, t}}$ can still be taken over. Only for finitely many $n$ it happens that the value $c=\varphi_{n}(0)$ is defined and that $c^{\infty}$ has no index in $\bigcup_{k \leq n} J_{k, a_{k}}$; only for these finitely many $n$ it happens that $\psi_{e_{n}}(0) \neq \varphi_{n}(0)$.

Each time $a_{n, t+1}>a_{n, t}$ during the construction, new constant functions are chosen for indices in $J_{n, a_{n, t+1}}$ so that each index in this interval belongs to

$$
\mathrm{SD}_{\psi, t}=\left\{e:(\forall j<e)\left[\psi_{j, t}(0) \neq \psi_{e, t}(0)\right]\right\} .
$$

Fix a stage $s$ and let $j \in J_{n, a_{n, s}}$. If for some $e_{k}<j$ we have that $\psi_{e_{k}, s}(0)$ first converges to $\psi_{j, s}(0)$ in stage $s$, then by the Kolmogorov complexity argument above, $a_{k}>a_{k, s}$ (except for finitely many $k$ ). As $n>k$, this means that also $a_{n}>a_{n, s}$, so $J_{n, a_{n, s}} \neq J_{n, a_{n}}$, whence we obtain from the converse $\mathrm{SD}_{\psi} \cap I_{n}=J_{n, a_{n}}$. Therefore $\mathrm{SD}_{\psi}=\bigcup_{n} J_{n, a_{n}}$ and (2.2) follows.

(II). A further property of the $\mathrm{SD}_{\psi}$ constructed is that $\mathrm{SD}_{\psi}$ is retraceable by a total function $f$, defined as follows: 
- If $x \in J_{n, k}-\left\{\min J_{n, k}\right\}$, then $f(x)=x-1$.

- If $x \in J_{0,1} \cup\left\{\min \left(J_{0,2}\right), e_{0}, e_{1}, e_{2}, \ldots\right\}$, then $f(x)=x$.

- Otherwise if $x=\min J_{n+1, k}$, where

$$
k=2^{n+1} b_{0}+2^{n} b_{1}+\ldots+2^{0} b_{n+1}+1
$$

for some $b_{0}, \ldots, b_{n} \in\{0,1\}$, then $f$ looks to the next level down. That is, $f(x)$ is defined to be the greatest member of $J_{n, k^{\prime}}$, where

$$
k^{\prime}=2^{n} b_{0}+2^{n-1} b_{1}+\ldots+2^{0} b_{n}+1 .
$$

Note that $f$ is everywhere defined. In the case where $x \in \mathrm{SD}_{\psi}$, then $x \in J_{n, a_{n}}$ for some $n$. It is vacuous to verify retraceability for $n=0$, so assume $n>0$. When $x=\min J_{n, a_{n}}$ then $f(x)=\max J_{n-1, a_{n-1}}$, otherwise $f(x)=x-1 \in$ $J_{n, a_{n}}$. In either case, $f(x)$ points to the next lesser element of $\mathrm{SD}_{\psi}$, hence $f$ is retraceable.

It is known from [16, comment before Theorem 30] that a set which is retraceable by a total recursive function is also $(1,2)$-recursive, that is, approximable. As the proof of this statement does not appear in the earlier paper [16], we give it here. We have already shown that $\mathrm{SD}_{\psi}$ is retraceable by a total function in part (II), so approximability follows from this fact.

Suppose $A$ is retraceable by a recursive function $g$. We define a recursive function $f$ which witnesses that $A$ is approximable (in fact, $(1,2)$-recursive) as follows. On input $(x, y)$ with $x<y$, iteratively compute $g(y), g \circ g(y), g \circ$ $g \circ g(y), \ldots$ until one of the following conditions holds:

- the next term increases or stays the same;

- the sequence drops below $x$.

If $x$ is found to be in this sequence then define $f(x, y)=(1,0)$ else define $f(x, y)=(0,0)$. If $f(x, y)=(1,0)$ and $A(y)=1$, then $A(x)=1$ because this sequence retraces down to all members of $A$ below $y$, including $x$. On the other hand, if the sequence did not retrace down to $x$, then it must have been the case that either $y \notin A$ or else $x \notin A$, so $f(x, y)=(0,0)$ agrees with the two-place characteristic function $A(x, y)$ in at least one coordinate.

This concludes the proof. 
Remark. Schaefer showed that $\operatorname{MIN}_{\varphi}$ is not $(1,2)$-recursive for any acceptable numbering $\varphi$ [25, Theorem 2.22]. In contrast, $\mathrm{SD}_{\varphi}$ can be $(1,2)$ recursive as was shown in part (II). We note that Kummer independently found an acceptable numbering $\varphi$ such that $\mathrm{SD}_{\varphi} \Varangle_{\mathrm{tt}} K$ using game-theoretic methods [14].

Figueira, Stephan and $\mathrm{Wu}[9]$ showed that there exists an infinite truth-table antichain of Chaitin's $\Omega$ 's. Since the set $\mathrm{SD}_{\psi}$ from Theorem 2.3 is truth-table equivalent to an arbitrarily chosen $\Omega$, we get the following result.

Corollary 2.4. There exist acceptable numberings $\psi^{[0]}, \psi^{[1]}, \ldots$ such that for all $i \neq j, \mathrm{SD}_{\psi^{[i]}} \not \mathbb{t t}_{\mathrm{tt}} \mathrm{SD}_{\psi^{[j]}}$.

There exist two acceptable numberings $\psi, \varphi$ with $\mathrm{SD}_{\psi}<_{\text {tt }} \mathrm{SD}_{\varphi}:$ Schaefer [25], [31] stated that there exists an acceptable numbering $\varphi$ where $\mathrm{SD}_{\varphi}$ is in the truth-table degree of $K$; Theorem 2.3 gives us an example of a further acceptable numbering $\psi$ where the truth-table degree of the set of the shortest descriptions is strictly below that of $K$. There are related open questions:

- Is there an infinite chain of truth-table degrees belonging to $\mathrm{SD}_{\psi}$ for various acceptable numberings $\psi$ ?

- Are there two different versions of Chaitin's $\Omega$ such that one is strictly truth-table below the other one? That is, can one construct the $\psi, \varphi$ with $\mathrm{SD}_{\psi}<_{\mathrm{tt}} \mathrm{SD}_{\varphi}$ using two different versions of $\Omega$ ?

In the year 1996, Kummer showed that the set of Kolmogorov non-random strings, given as $\{x: C(x)<\log x\}$, is truth-table complete for all Kolmogorov numberings $\varphi$. On the other hand, if one generalizes the definition of this set to acceptable numberings, there exists an acceptable numbering $\varphi$ for which this set is truth-table incomplete [15]. Since there exists a Kolmogorov numbering $\varphi$ such that $\mathrm{SD}_{\varphi} \equiv_{\text {tt }} K$ [31], we are left with an analogous problem for the set of shortest descriptions:

Question 2.5. Is $\mathrm{SD}_{\varphi} \geq_{\text {tt }} K$ for every Kolmogorov numbering $\varphi$ ?

The canonical universal Turing machine is a Kolmogorov numbering, so a positive answer to this question would identify a uniform property among the most familiar programming languages.

Proposition 2.6. For any (not necessarily acceptable) numbering $\psi$ of the partial recursive functions, $\mathrm{SD}_{\psi}$ is a left-r.e. set. Furthermore, the complement of $\mathrm{SD}_{\psi}$ is not immune. 
Proof. Let $\mathrm{SD}_{\psi, s}$ denote the set of indices which appear to be shortest descriptions after time $s$. Then for any $t>s, \mathrm{SD}_{\psi, s}<_{\text {lex }} \mathrm{SD}_{\psi, t}$. Indeed an index only ever changes from a shortest description to a non-shortest description if some smaller index takes over its value.

Note that there is a strictly ascending recursive sequence of indices $e_{0}, e_{1}, \ldots$ such that $\psi_{e_{n}}(0) \downarrow=0$ and $\psi_{e_{n}}(1) \downarrow=n$; this sequence can be defined by letting $e_{n}$ be the first index found when searching by dovetailing for an index with these two properties. Then $\left\{e_{1}, e_{2}, \ldots\right\}$ is an infinite recursive set which is disjoint to $\mathrm{SD}_{\psi}$, hence the complement of $\mathrm{SD}_{\psi}$ is not immune.

While $\mathrm{SD}_{\psi}$ is immune for acceptable numberings [25], somehow immunity is not guaranteed for all numberings. Let $\varphi$ be any acceptable numbering. Then the following $K$-acceptable numbering satisfies that $\mathrm{SD}_{\psi}$ is recursive:

$$
\psi_{\langle x, e\rangle}(z)= \begin{cases}\uparrow & \text { if } x=0 \text { and } z=0, \\ x-1 & \text { if } x>0 \text { and } z=0, \\ \varphi_{e}(z) & \text { if } z>0 .\end{cases}
$$

For this numbering, $\mathrm{SD}_{\psi}=\{\langle 0,0\rangle,\langle 1,0\rangle,\langle 2,0\rangle, \ldots\}$ with an appropriately defined pairing function. The numbering is $K$-acceptable as the oracle $K$ permits to determine which $x$ has to be chosen such that $\psi_{\langle x, y\rangle}=\varphi_{y}$. In addition, we also get the following annoying result:

Proposition 2.7. For any r.e. set $A$, there exists a $K$-acceptable numbering $\nu$ such that $\mathrm{SD}_{\nu} \equiv_{\mathrm{btt}} A$.

Proof. Let $\varphi_{e}$ be any acceptable numbering. Let

$$
\psi_{e}(x)= \begin{cases}\varphi_{e}(x) & \text { if } x>0, \\ \varphi_{e}(0) & \text { if }\left[x=0 \quad \& \quad \varphi_{e}(0) \downarrow \leq e\right], \\ \uparrow & \text { otherwise. }\end{cases}
$$

Note that $\psi$ is a $K$-acceptable numbering, as $\varphi$ has arbitrarily large indices for each function by the Padding Lemma [27]. Now define $\nu$ so as to satisfy:

- $\nu_{3 e}(0)= \begin{cases}e & \text { if } e \in A, \\ \uparrow & \text { otherwise, }\end{cases}$

- $\nu_{3 e+1}(0)=e$ and

- $\nu_{3 e+2}=\psi_{e}$. 
Then $3 e+2 \notin \mathrm{SD}_{\nu}$ for all $e$ and whenever $e \neq \min \bar{A}$,

$$
3 e \in \mathrm{SD}_{\nu} \Longleftrightarrow e \in A \Longleftrightarrow 3 e+1 \notin \mathrm{SD}_{\nu}
$$

Thus $\mathrm{SD}_{\nu} \equiv_{\text {btt }} A$ and $\nu$ inherits the property of being $K$-acceptable from $\psi$.

Hence, the study of $\mathrm{SD}_{\psi}$ does not generalize in an interesting way to numberings which are not acceptable. We summarize the known properties for the set of shortest descriptions in Corollary 2.8.

Corollary 2.8. There exists an acceptable numbering $\psi$ such that the set $\mathrm{SD}_{\psi}$ is:

(I) $\omega$-immune but not hyperimmune or bi-immune,

(II) wtt-complete but not tt-complete,

(III) retraceable and approximable,

(IV) 2-r.e. and left-r.e. but not co-2-r.e.

Proof. Similarly to the case for $\operatorname{MIN}_{\varphi}$ [1], [20], Schaefer argued that $\mathrm{SD}_{\varphi}$ is $\omega$-immune but not hyperimmune for every acceptable numbering $\varphi$ [25]. Furthermore, $\mathrm{SD}_{\varphi}$ is 2-r.e. but not co-2-r.e. [31] and wtt-complete [25] for every acceptable numbering $\varphi$. Let $\psi$ be the special acceptable numbering given by Theorem 2.3. Then $\mathrm{SD}_{\psi}$ is a left-r.e. set satisfying (I)-(IV) by Proposition 2.6.

If one is not interested in shortest descriptions, one could more directly construct a set satisfying the four properties above. The set constructed in Corollary 2.8 is truth-table equivalent to $\Omega$, hence

$$
\{\sigma: \sigma \text { is a prefix of } \Omega\}
$$

satisfies the properties (I)-(IV) of the corollary. Property (IV) from Corollary 2.8 in fact holds in every acceptable numbering of the set of shortest descriptions [31] and so for any acceptable $\varphi, \mathrm{SD}_{\varphi}$ btt-reduces to the halting set but does not many-one reduce to the halting set. This contrasts with the set of Kolmogorov random strings and the set of shortest programs which many-one reduce to $\bar{K}$ and $K^{\prime}$ respectively.

\section{Domain-random strings}

In a letter dated February 2002 [10], Friedman introduced the set of domainrandom strings, the complement of

$$
\mathrm{NRW}_{\varphi}=\left\{x:(\exists j<x)\left[\max \left(W_{j}^{\varphi} \cup\{0\}\right)=x\right]\right\},
$$


which is a variant of the set of Kolmogorov non-random strings. In his letter, Friedman pointed out that the set has some properties similar to the set of shortest descriptions and asked for the Turing degree of $\mathrm{NRW}_{\varphi}$. Three months later, Davie announced that $\mathrm{NRW}_{\varphi} \equiv_{\mathrm{T}} K$ for all Kolmogorov numberings $\varphi[6]$ and noted, under advisement from Solovay, that his argument could be generalized to cover the acceptable numberings with polynomiallybounded translation function. Stephan independently published a proof of Davie's result, based on the Owings Cardinality Theorem, for numberings satisfying the Kolmogorov property [29]. He adds that $\mathrm{NRW}_{\varphi}$ is never 2-r.e. for any acceptable numbering $\varphi$, but notes that there exists an acceptable numbering $\psi$ such that $\mathrm{NRW}_{\psi}$ is the complement of a 2-r.e. set.

While the Kolmogorov property helps us to fix the Turing degree for the set of domain-random strings, for stronger reductions this property counts against us. Indeed for any Kolmogorov numbering $\varphi, \mathrm{NRW}_{\varphi}$ is not $n$ r.e. for any $n$ [29]; hence $\mathrm{NRW}_{\varphi} \mathbb{Z}_{\text {btt }} K$ for Kolmogorov numberings $\varphi$. As in the case for the set of shortest descriptions and the set of shortest programs, $K \not_{\mathrm{btt}} \mathrm{NRW}_{\psi}$ for all acceptable numberings $\psi$ (because $\mathrm{NRW}_{\psi}$ is $\omega$-immune, see [8], [25]). Since $\mathrm{NRW}_{\psi} \leq_{\mathrm{tt}} K$ for every numbering $\psi$ (as $\mathrm{NRW}_{\psi}$ is $\omega$-r.e.), $\mathrm{NRW}_{\varphi}$ is a natural example of a set which is bttincomparable with the halting set and truth-table reducible to it whenever $\varphi$ is a Kolmogorov numbering.

While for a general acceptable numbering $\varphi$ the Turing degree of $\mathrm{NRW}_{\varphi}$ is unresolved, we have the following result for truth-table degrees.

Proposition 3.1. There exist acceptable numberings $\psi$ and $\nu$ such that

(I) $\mathrm{NRW}_{\psi} \equiv_{\mathrm{tt}} K$ and

(II) $\mathrm{NRW}_{\nu} \nsupseteq_{\mathrm{tt}} K$.

Proof. (I). Let $\varphi$ be an acceptable numbering. Define the acceptable numbering $\psi$ as follows:

- $\operatorname{dom} \psi_{0}=\emptyset$.

- If $e=5^{x}$ for some $x$ then let $\psi_{e}=\varphi_{x}$,

- otherwise $e=5^{x}+k$ for some $x$ and $k \in\left\{0,1, \ldots, 4 \cdot 5^{x}-1\right\}$. In this case define $\psi_{e}$ such that

$$
\operatorname{dom} \psi_{e}= \begin{cases}\{e+1\} & \text { if } x \in K, \\ \emptyset & \text { if } x \notin K .\end{cases}
$$


Let

$$
A_{x}=\left\{5^{x}+2,5^{x}+3, \ldots, 5^{x+1}\right\} .
$$

Now it is shown for $x>0$ that

$$
x \in K \Longleftrightarrow A_{x} \subseteq \mathrm{NRW}_{\psi} .
$$

If $x \notin K$, then $e \notin \mathrm{NRW}_{\psi}$ for some few $e \in A_{x}$. Furthermore, there are $5^{x+1}-\left(5^{x}+1\right)=4 \cdot 5^{x}-1$ indices in $A_{x}$, which is more than the number of lesser indices in $\left\{0,1, \ldots, 5^{x}\right\}$.

If $x \in K$, then $W_{e}^{\psi}=\{e+1\}$ for $e=5^{x}+1,5^{x}+2, \ldots, 5^{x+1}-1$. In other words, $\max \left(W_{e}^{\psi} \cup\{0\}\right)=e+1$ for all $e$ with $e+1 \in A_{x}$. So $A_{x+1} \subseteq \mathrm{NRW}_{\psi}$.

(II). Our goal here will be to ensure that the domain-random strings have high Kolmogorov complexity. Fix an acceptable numbering $\varphi$, let $a_{n}$ be as in $(2.1)$, let

$$
b_{n}=2^{n+2}-a_{n},
$$

and let $\left\{b_{n, t}\right\}$ be a decreasing approximation to $b_{n}$. Let

$$
I_{n}=\left\{2^{n+1}+1,2^{n+1}+2, \ldots, 2^{n+2}-1\right\}
$$

for all $n$ and note that $b_{n} \in I_{n}$ for all but finitely many $n$. We define an acceptable numbering $\psi$ so as to satisfy the following two conditions:

- for all $n, \nu_{2^{n}}=\varphi_{n}$;

- for all but finitely many $n, \overline{\mathrm{NRW}}_{\nu} \cap I_{n+1}=\left\{b_{n}\right\}$.

It is obvious how to achieve the first point: just define $\nu$ in the manner prescribed. To satisfy the second point, we define for all $x \in I_{n}$ :

$$
W_{x}^{\nu}= \begin{cases}\{x+1, x+2\} & \text { if } x+1 \geq b_{n}, \\ \{x+1\} & \text { otherwise }\end{cases}
$$

In more detail, $W_{x, 0}^{\nu}=\{x+1\}$ for all $x \in I_{n}$ and at each stage $t+1$ where $b_{n, t+1}<b_{n, t}$ for some $n$, we set

$$
W_{x, t+1}^{\nu}=\{x+1, x+2\} \text { for all } x \in\left\{b_{n, t+1}-1, b_{n, t+1}, \ldots, 2^{n+1}-1\right\} .
$$

This puts $b_{n, t+1}$ into the $(t+1)^{\mathrm{st}}$ stage of $\overline{\mathrm{NRW}}_{\nu}$ while the rest of the numbers in $I_{n}$ remain in $\mathrm{NRW}_{\nu}$ forever unless $b_{n, s}<b_{n, t+1}$ for some $s>t+1$. Finally, we assign some arbitrary function to $\nu_{0}$. 
It remains to demonstrate that the indices of the form $\nu_{2}$ do not destroy the second point above. Since $\Omega$ is Martin-Löf random, we have $C\left(b_{n}\right)>n / 2$ for all but finitely many $n$. If some $m$ with $2^{m}<b_{n}$ satisfies $\max \left(W_{2^{m}}^{\nu} \cup\right.$ $\{0\})=b_{n}$ then

$$
C\left(b_{n}\right) \leq 2 \log n+2 \log m+O(1) \leq 4 \log n+O(1),
$$

but this can only happen finitely often. Since $\Omega$ does not tt-compute the halting set [2], we get

$$
\overline{\mathrm{NRW}}_{\nu}={ }^{*}\left\{b_{0}, b_{1}, b_{2}, \ldots\right\} \equiv_{\mathrm{tt}} \Omega \geq_{\mathrm{tt}} K .
$$

This concludes the overall proof.

We note that the truth-table incomplete version of $\overline{\mathrm{NRW}}_{\nu}$ in Proposition 3.1 inherits properties (I)-(IV) from Corollary 2.8: it is wtt-complete, retraceable, approximable, 2-r.e., and non-hyperimmune. The wtt-completeness of $\overline{\mathrm{NRW}}_{\nu}$ follows from the fact that $\Omega$ is wtt-complete [2]. Stephan [29] showed for every acceptable numbering $\varphi$ that $\mathrm{NRW}_{\varphi}$ is not 2-r.e.: the main reason is that $\mathrm{NRW}_{\varphi}$ is neither co-r.e. nor properly 2 -r.e. as $\overline{\mathrm{NRW}}_{\varphi}$ is immune. The proof that $\overline{\mathrm{NRW}}_{\varphi}$ is $\omega$-immune is given below in Proposition 3.5.

Remark. The numbering $\psi$ in Proposition 3.1(I) can be upgraded to a Kolmogorov numbering via the construction [25, Theorem 2.17]. It therefore follows from the comments preceding Proposition 3.1 that $\mathrm{NRW}_{\psi}$ is truthtable complete but btt-incomparable to the halting set for this upgraded $\psi$.

This observation leads us to the following question:

Question 3.2. Is $\mathrm{NRW}_{\varphi} \equiv_{\mathrm{tt}} K$ for every Kolmogorov numbering $\varphi$ ?

Stephan [28] showed that every nonrecursive truth-table degree contains an infinite antichain of btt-degrees. We show that for certain truth-table degrees such an antichain can be chosen as a set of domain-random strings. The following corollary subsumes the result from Proposition 3.1 because $K \not_{\mathrm{tt}} \Omega[2]$.

Theorem 3.3. Let $A$ be any r.e. set satisfying $\Omega \leq_{\mathrm{tt}} A \leq_{\mathrm{tt}} K$. There exist acceptable numberings $\psi^{[0]}, \psi^{[1]}, \psi^{[2]}, \ldots$ such that for all recursive $W_{d}$ and $W_{e}$,

(I) $\mathrm{NRW}_{\psi[d]} \leq_{\mathrm{btt}} \mathrm{NRW}_{\psi[e]} \Longleftrightarrow W_{d} \subseteq^{*} W_{e}$ and

(II) $\mathrm{NRW}_{\psi^{[e]}} \equiv_{\mathrm{tt}} A$. 
Here $W_{0}, W_{1}, \ldots$ is any acceptable numbering of the r.e. sets.

Proof. (I). We shall construct a set whose members are spread out sufficiently sparse and randomly that any self-membership query cannot obtain non-trivial information by querying larger indices of the set. Randomness will also prevent smaller indices from being useful for membership queries. For readability, let $\mathrm{NRW}_{e}$ denote $\mathrm{NRW}_{\psi^{[e]}}$. For each $e, \overline{\mathrm{NRW}}_{e}$ will look sufficiently similar to both this set and $W_{e}$ so as to satisfy (I).

Define a recursive function $h$ by

$$
\begin{aligned}
h(0) & =0, \\
h(n+1) & =2^{2 h(n)+3 n+2}+1
\end{aligned}
$$

and let

$$
g(n)=b_{4 h(n)+5 n}
$$

where $b_{n}$ is defined in (3.1). This pair of functions satisfies

$$
h(0)<g(0)<h(1)<g(1)<h(2)<g(2) \ldots
$$

so that the values of $g$ and $h$ alternate. Let $\varphi$ be any acceptable numbering. We shall define uniformly in $e$ a numbering $\psi^{[e]}$ in such a way that

$$
\overline{\mathrm{NRW}}_{e}={ }^{*}\{g(3 n): n \in \mathbb{N}\} \cup\left\{g(3 n+1): n \in W_{e}^{\varphi}\right\} \cup\{g(3 n+2): n \in A\} .
$$

This can be done as follows. Define $\psi_{h(3 e)}=\varphi_{e}$ for all $e$. Since $h$ is recursive, this ensures acceptability. The remaining $\psi$-indices are defined so as to 
satisfy:

$$
W_{x}^{\psi^{[e]}}= \begin{cases}\{x+1\} & \text { if } h(3 n)<x<g(3 n)-1, \\ \{x+1, x+2\} & \text { if } g(3 n)-1 \leq x<g(3 n+1)-2, \\ \{x+1, x+2\} & \text { if } g(3 n+1)-2 \leq x<g(3 n+2)-2 \\ & \text { and } x \notin W_{e}^{\varphi}, \\ \{x+1, x+2, x+3\} & \text { if } g(3 n+1)-2 \leq x<g(3 n+2)-3 \\ & \text { and } x \in W_{e}^{\varphi}, \\ \{x+1, x+2, x+3\} & \text { if } g(3 n+2)-3 \leq x<h(3 n+3), \\ \{x+1, x+2, x+3, x+4\} & \text { and } x \notin A \text { and } x \in W_{e}^{\varphi}, \\ & \text { and } g(3 n+2)-3 \leq x<h(3 n+3), \\ \{x+1, x+2\} & \text { if } g(3 n+2)-2 \leq x<h(3 n+3), \\ & \text { and } x \notin A \text { and } x \notin W_{e}^{\varphi}, \\ \{x+1, x+2, x+3\} & \text { if } g(3 n+2)-2 \leq x<h(3 n+3), \\ & \text { and } x \in A \text { and } x \notin W_{e}^{\varphi} .\end{cases}
$$

Since $g$ is approximable from above, the functions $\psi_{0}^{[e]}, \psi_{1}^{[e]}, \ldots$ are indeed partial recursive. Similar to (3.2) we have that for all but finitely many $n$,

$$
\max \left(W_{h(k)}^{\psi^{[e]}} \cup\{0\}\right) \neq g(n)
$$

whenever $k \leq n$, and therefore (3.4) holds. $\psi$-indices $h(3 n+1)$ and $h(3 n+2)$ have no special purpose in this construction beyond satisfying (3.3).

The reverse direction of Theorem 3.3(I) is now immediate. If $W_{d}^{\varphi} \subseteq^{*}$ $W_{e}^{\varphi}$ and these sets are recursive, then $\mathrm{NRW}_{d}=\mathrm{NRW}_{e}$, except possibly on intervals $I_{n}$ of the form

$$
I_{n}=\{h(3 n+1), h(3 n+1)+1, \ldots, h(3 n+2)\} .
$$

Let $a \in \mathrm{NRW}_{e}$ and define a recursive function $f$ by

$$
f(x)= \begin{cases}x & \text { if } x \notin I_{n} \text { for some } n, \\ a & \text { if } x \in I_{n} \text { and } x \notin W_{d}^{\varphi}, \\ x & \text { if } x \in I_{n} \text { and } x \in W_{d}^{\varphi}\end{cases}
$$

Then $x \in \mathrm{NRW}_{d}$ iff $f(x) \in \mathrm{NRW}_{e}$ for all but finitely many $x$. So in fact we get $\mathrm{NRW}_{d} \leq_{\mathrm{m}} \mathrm{NRW}_{e}$. 
For the forward direction, assume for a contradiction that there exist infinitely many $n$ such that $n \in W_{d}^{\varphi}-W_{e}^{\varphi}$. First we show that if there is a btt-reduction $F$ witnessing $\mathrm{NRW}_{d} \leq_{\text {btt }} \mathrm{NRW}_{e}$, then there is a further bttreduction $R$ which determines membership in $I_{n} \cap \mathrm{NRW}_{d}$ without querying $\psi^{[e]}$-indices larger than $\max I_{n}$. The reason for this is that all queries from $F$ to $\mathrm{NRW}_{e} \cap\left\{x: x \geq \max I_{n}\right\}$ return the answer "1," and therefore all such replies can be hard-coded into the recursive function of $R$. Indeed the only non-zero places in the characteristic function of $\mathrm{NRW}_{e}$ occur in the range of $g$, and by choice of $g$ we have

$$
C[g(n)] \geq 2 h(n)+\frac{5}{2} n
$$

for all $n$. Since the queries for $F$ are a recursive function of their input, we have that any $F$-query with input in $I_{n}$ has Kolmogorov complexity at most $\log h(3 n+2)+O(1)$ and therefore cannot query among $\{g(3 n+2)$, $g(3 n+3), \ldots\}$ except finitely often. Thus our btt-reduction $R$ does not query above $\max I_{n}$ to decide membership in $\mathrm{NRW}_{d} \cap I_{n}$ for sufficiently large $n$, say $n>N$.

We now argue that the $\psi^{[e]}$-indices below $\max I_{n}$ do not contain sufficient information for $R$ to determine membership in $\mathrm{NRW}_{d} \cap I_{n}$ whenever $n \in$ $W_{d}^{\varphi}-W_{e}^{\varphi}$ and $n>N$. For all such $n$, the set $\operatorname{NRW}_{d} \cap\left\{x: x \leq \max I_{n}\right\}$ can be computed given $d, e, R, n, A \| n$, and $g(3 n)$ because $I_{n} \cap \mathrm{NRW}_{e}=\emptyset$. In particular, $g(3 n+1)$ can be computed using these components as it is the unique nonmember of $\mathrm{NRW}_{d} \cap I_{n}$. It follows that

$$
C[g(3 n+1)] \leq 2[n+g(3 n)]+O(1) \leq 2 h(3 n+1)+2 n+O(1),
$$

contradicting (3.5) for all but finitely many $n$. Therefore no such reduction $R$ exists, and we conclude $\mathrm{NRW}_{d} \not \underline{\mathrm{btt}}_{\mathrm{t}} \mathrm{NRW}_{e}$.

(II). Since

$$
n \in A \Longleftrightarrow \mathrm{NRW}_{e} \cap\{h(3 n+2), h(3 n+2)+1, \ldots, h(3 n+3)\} \neq \emptyset,
$$

we have $A \leq_{\mathrm{tt}} \mathrm{NRW}_{e}$. On the other hand, since $W_{e}$ is recursive, we get $\mathrm{NRW}_{e} \leq_{\mathrm{tt}} A \oplus \Omega \equiv_{\mathrm{tt}} A$.

This completes the proof.

We leave open the question of whether such btt-chains and antichains can be constructed using Kolmogorov numberings in place of acceptable ones and whether similar btt properties can be achieved using the set of shortest 
descriptions or even the set of shortest programs. We note that Kinber's construction [13] which gives a pair of acceptable numberings with distinct bounded truth-table degrees for the set of shortest programs does not make use of Kolmogorov complexity.

Corollary 3.4. Every r.e. truth-table degree between the degree of $\Omega$ and the degree of $K$ contains infinite ascending chains, infinite descending chains, and infinite antichains of btt-degrees.

Proof. Let

$$
P_{n}=\left\{p_{n}, p_{n}^{2}, p_{n}^{3}, \ldots\right\}
$$

be the set powers of the $n^{\text {th }}$ prime number. Then applying Theorem 3.3 to the sequence of recursive sets $P_{1}, P_{2}, P_{3}, \ldots$ yields an antichain of bttdegrees. Let

$$
U_{n}=\bigcup_{k \leq n} P_{n}
$$

Then $U_{1}, U_{2}, \ldots$ gives rise to an ascending chain, and $\overline{U_{1}}, \overline{U_{2}}, \ldots$ gives rise to a descending chain.

As was the case in Corollary 2.4, it is also possible to construct a collection of acceptably-numbered NRW's which form a truth-table degree antichain.

For comparison with the set of shortest descriptions, we now discuss immunity for the set of domain-random strings. We referenced the following fact earlier, and for completeness we now give a proof.

Proposition 3.5. For every acceptable numbering $\varphi, \overline{\mathrm{NRW}}_{\varphi}$ is $\omega$-immune.

Proof. Suppose $\overline{\mathrm{NRW}}_{\varphi}$ is not $\omega$-immune. Let $f$ be a recursive function such that $D_{f(i)} \cap \overline{\mathrm{NRW}}_{\varphi} \neq \emptyset$ for all $i$ where $D_{f(i)}$ is a uniform collection of pairwise disjoint sets each with cardinality $k$. Using the $s-m$ - $n$ Theorem, define recursive functions $g_{i}$ by letting $\varphi_{g_{i}\left(x_{1}, \ldots, x_{k}\right)}(z)$ be the $i^{\text {th }}$ member of $D_{f(z)}$ if $z$ is the least index satisfying $\min D_{f(z)}>\max \left\{x_{1}, \ldots, x_{k}\right\}$; otherwise $\varphi_{g_{i}\left(x_{1}, \ldots, x_{k}\right)}(z)$ is undefined.

By the Recursion Theorem, there exist indices $e_{1}, \ldots, e_{k}$ such that $\varphi_{e_{i}}=$ $\varphi_{g_{i}\left(e_{1}, \ldots, e_{k}\right)}$ for all $i$. Let $y_{i}$ be the unique element in the range of $\varphi_{e_{i}}$. For at least one $i, y_{i}$ is a member of $\overline{\mathrm{NRW}}_{\varphi}$ by assumption on $f$. On the other hand, $y_{i} \in \mathrm{NRW}_{\varphi}$ by definition of $g_{i}$, a contradiction.

A set which is $\omega$-immune avoids the btt-cone above the halting set [8] and therefore:

Corollary 3.6. $\mathrm{NRW}_{\varphi} \Varangle_{\mathrm{btt}} K$ for all acceptable numberings $\varphi$. 
A set is called $\Pi_{1}$-immune if it contains no infinite co-r.e. subset. In contrast to the set of shortest descriptions, where $\Pi_{1}$-immunity depends on the underlying acceptable numbering, $\overline{\mathrm{NRW}}_{\varphi}$ is $\Pi_{1}$-immune for every acceptable numbering $\varphi . \Pi_{1}$-immunity for the set of shortest descriptions varies for the same reason as for the set of shortest programs [30].

Theorem 3.7. For every acceptable numbering $\varphi, \overline{\mathrm{NRW}}_{\varphi}$ is $\Pi_{1}$-immune.

Proof. Let

$$
f(x)=\max _{e \leq x} \varphi_{e}(x)+1
$$

where this maximum is taken over all functions which converge. Now $f \leq_{\mathrm{T}}$ $K$, hence there exists a recursive approximation $f_{0}, f_{1}, \ldots$ which converges to $f$. Using the $s-m-n$ Theorem, define a recursive function $g$ by

$$
x \in W_{g(e)}^{\varphi} \quad \Longleftrightarrow \quad(\exists s)(\forall y<x)\left[x \leq f_{s}(e) \quad \vee \quad y \in W_{e, s}^{\varphi}\right] .
$$

Now for almost all $e, g(e) \leq f(e)$. It follows that for almost all $e$ where $W_{e}^{\varphi}$ is coinfinite that the maximum of $W_{g(e)}^{\varphi}$ exists, is larger than $g(e)$, and is a nonmember of the set $W_{e}^{\varphi}$. Therefore every infinite co-r.e. set intersects $\mathrm{NRW}_{\varphi}$

On the other hand, $\mathrm{NRW}_{\varphi}$ itself is not immune for any Kolmogorov numbering $\varphi$.

Theorem 3.8. Let $\psi$ be a numbering of the partial-recursive functions. If $\psi$ has the Kolmogorov property or $\psi$ is acceptable, then $\mathrm{NRW}_{\psi}$ contains an infinite recursive subset.

Proof. First let us consider the case where $\psi$ has the Kolmogorov property. For each $n$ let $A_{n}$ denote the singleton set $\left\{n^{2}\right\}$, and let $f$ be a translation function from an enumeration $A_{0}, A_{1}, \ldots$ into $\psi$ with linear bound $c$. Then for all but finitely many $n$,

$$
f(n) \leq c n+c<n^{2}
$$

and

$$
\max \left(W_{f(n)}^{\psi} \cup\{0\}\right)=n^{2},
$$

hence $n^{2} \in \mathrm{NRW}_{\psi}$ for all but finitely many $n$.

Now suppose that $\psi$ is an acceptable numbering. Using the $s-m-n$ Theorem, define a recursive function $f$ satisfying $W_{f(x, c)}^{\psi}=\{x+c\}$. By the 
Recursion Theorem with Parameters [27], there exists a recursive function $n$ such that

$$
W_{n(c)}^{\psi}=W_{f[n(c), c]}^{\psi}=\{n(c)+c\},
$$

and therefore $n(c)+c \in \mathrm{NRW}_{\psi}$ for all $c$. Moreover, $\{n(c)+c: c \in \mathbb{N}\}$ is an infinite r.e. set and thus must contain an infinite recursive subset of $\mathrm{NRW}_{\psi}$.

An analogous argument shows that both $\overline{\operatorname{MIN}}_{\varphi}$ and $\overline{\mathrm{SD}}_{\varphi}$ both contain infinite recursive subsets in any numbering $\varphi$ with the Kolmogorov property. Since every acceptable numbering contains infinitely many indices for each function, it follows immediately that $\overline{\mathrm{MIN}}_{\psi}$ and $\overline{\mathrm{SD}}_{\varphi}$ contain infinite recursive subsets in every acceptable numbering $\psi$.

Finally we remark that the following holds for every acceptable numbering $\varphi$ : although $\overline{\mathrm{NRW}}_{\varphi}$ is both immune and $\Pi_{1}$-immune, $\overline{\mathrm{NRW}}_{\varphi}$ does contain an infinite left-r.e. subset.

Theorem 3.9. For every acceptable numbering $\varphi, \overline{\mathrm{NRW}}_{\varphi}$ has an infinite left-r.e. subset.

Proof. Using the Padding Lemma [27], define an increasing recursive function $f$ so that $f(0)=0$ and at least $f(e)+1 \varphi$-indices for the empty function exist below $f(e+1)$ for all $e \geq 1$. At Stage 0 we define our left-r.e. set to have the characteristic function

$$
0^{f(0)-1} 10^{f(1)-1} 10^{f(2)-1} 1 \ldots
$$

We denote the current positions of the 1's at each stage by $a_{0}, a_{1}, a_{2}$ with $a_{0}<a_{1}<a_{2}<\ldots$ so that initially $a_{n}=f(n)$ for all $n$. If at some stage $a_{n}$ appears to be in $\mathrm{NRW}_{\varphi}$, then we update our left-r.e. set with $a_{n}=a_{n}-1$. This can only happen $f(n+1)-f(n)-1$ times for any $n$ by choice of $f$. Hence $f(n)<a_{n} \leq f(n+1)$ for all stages and $a_{n} \in \overline{\mathrm{NRW}}_{\varphi}$ in the limit.

\section{Turing-minimal indices}

In spring 1990 (according to the best recollection of the author), Case [3] introduced a variant of the set of shortest programs within a homework assignment:

$$
\operatorname{MIN}_{\varphi}^{*}=\left\{e:(\forall j<e)\left[W_{j}^{\varphi} \neq^{*} W_{e}^{\varphi}\right]\right\} .
$$

Schaefer [25] investigated this variant and showed that there exists a Kolmogorov numbering $\varphi$ such that $\operatorname{MIN}_{\varphi}^{*}$ is truth-table equivalent to $K^{\prime \prime}$. 
Teutsch [31] [32] added that a Kolmogorov numbering $\psi$ can simultaneously satisfy $\mathrm{MIN}_{\psi}^{*} \equiv_{\mathrm{tt}} K^{\prime \prime}$ and

$$
\operatorname{MIN}_{\psi}^{\mathrm{m}}=\left\{e:(\forall j<e)\left[W_{j}^{\psi} \not \equiv_{\mathrm{m}} W_{e}^{\psi}\right]\right\} \equiv_{\mathrm{tt}} K^{\prime \prime} .
$$

Definition 4.1. Define the set

$$
\operatorname{MIN}_{\varphi}^{\mathrm{T}^{(n)}}=\left\{e:(\forall j<e)\left[\left(W_{j}^{\varphi}\right)^{(n)} \not_{\mathrm{T}}\left(W_{e}^{\varphi}\right)^{(n)}\right]\right\}
$$

where ${ }^{(n)}$ denotes the $n^{\text {th }}$ iteration of the jump operator.

It is known that $\operatorname{MIN}_{\varphi}^{*} \equiv_{\mathrm{T}} \operatorname{MIN}_{\varphi}^{\mathrm{m}} \equiv_{\mathrm{T}} K^{\prime \prime}$ and $\operatorname{MIN}_{\varphi}^{\mathrm{T}} \equiv_{\mathrm{T}} K^{\prime \prime \prime}$ whenever $\varphi$ is a Kolmogorov numbering [12], yet the truth-table degrees for these sets under Kolmogorov numberings is not known in general. For the more general case of acceptable numberings $\varphi$, even the Turing degrees of $\mathrm{MIN}_{\varphi}^{*}$, $\operatorname{MIN}_{\varphi}^{\mathrm{m}}$, and $\mathrm{MIN}_{\varphi}^{\mathrm{T}}$ are unknown [31]. By the familiar result of Fenner and Schaefer [8], [25], each of these sets avoids the btt-cone above the halting set. The following answers a question left from [31].

Theorem 4.2. There exists a Kolmogorov numbering $\psi$ such that for all $n$, $\operatorname{MIN}_{\psi}^{\mathrm{T}^{(n)}} \equiv_{\mathrm{tt}} \emptyset^{(n+4)}$.

It is interesting to note that Theorem 4.2 uses infinite injury in the case $n=0$ whereas the equivalent result for Turing degrees, $\mathrm{MIN}^{\mathrm{T}} \equiv_{\mathrm{T}} K^{\prime \prime \prime}$, requires only finite injury [31]. Below are the results used to prove Theorem 4.2. The Sack Density Theorem, the Robinson Interpolation Theorem, and the Sacks Jump Theorem are all used. It is worth noting that Schwarz's proof of Proposition 4.7 also makes use of infinite injury by applying the Yates Index Set Theorem.

Theorem 4.3 (Sacks Density Theorem [24]). Let $B$ and $C$ be r.e. sets with $B<_{\mathrm{T}} C$. Then there exists a r.e. set $D$ such that $B<_{\mathrm{T}} D<_{\mathrm{T}} C$.

The following theorem appears as "Corollary 4" in [22]:

Theorem 4.4 (Robinson [22]). Let $B$ and $C$ be r.e. sets with $B \leq_{\mathrm{T}} C$, and let $A_{0}, A_{1}, \ldots$ be a sequence of r.e. sets satisfying $A_{i} \leq_{\mathrm{T}} C$ and $C \mathbb{Z}_{\mathrm{T}} A_{i} \mathbb{Z}_{T}$ $B$ for all $i$. Then there exists a r.e. set $D$ such that $B \leq_{\mathrm{T}} D \leq_{\mathrm{T}} C$ and $\left.D\right|_{\mathrm{T}} A_{i}$ for all $i$. Furthermore, $D$ can be found uniformly from indices for $B, C$ and $\left\{A_{i}\right\}$.

Theorem 4.5 (Sacks Jump Theorem [23]). Let $B$ be any set, and let $S$ be r.e. in $B^{\prime}$ with $B^{\prime} \leq_{\mathrm{T}} S$. Then there exists a $B$-r.e. set $A$ with $A^{\prime} \equiv_{\mathrm{T}} S$. Furthermore, an index for $A$ can be found uniformly from an index for $S$. 
Definition 4.6. $\mathrm{HIGH}^{n}=\left\{e: W_{e}^{(n)} \equiv_{\mathrm{T}} K^{(n)}\right\}$.

Theorem 4.7 (Schwarz [26]). $\mathrm{HIGH}^{n}$ is $\Sigma_{n+4}$-complete.

Corollary 4.8. For any acceptable numbering $\varphi$, there exists a recursive function $f$ such that

- $e \in \operatorname{HIGH}_{\varphi}^{n} \Longrightarrow(\forall k)\left[f(e, k) \in \mathrm{HIGH}_{\varphi}^{n}\right]$

- $e \notin \operatorname{HIGH}_{\varphi}^{n} \Longrightarrow(\forall i \neq j)\left[\left.W_{f(e, i)}^{\varphi}\right|_{\mathrm{T}} W_{f(e, j)}^{\varphi}\right]$

Furthermore, $W_{f(e, k)}^{\varphi} \geq_{\mathrm{T}^{(n)}} W_{e}^{\varphi}$ for all $k$.

Proof. For simplicity of notation, we shall use $W_{e}$ in place of $W_{e}^{\varphi}$ in this proof. We first consider the case $n=0$. Define $f(e, 0)$ to be the index of a set obtained from Theorem 4.3 with $W_{e}$ being the lower bound and $K$ being the upper bound. An inspection of the algorithm from the proof of Theorem 4.3 reveals that $W_{f(e, 0)} \geq_{\mathrm{T}} W_{e}$ even if $W_{e} \equiv_{\mathrm{T}} K$. Thus

- $W_{e}<_{\mathrm{T}} K \Longrightarrow W_{e}<_{\mathrm{T}} W_{f(e, 0)}<_{\mathrm{T}} K$ and

- $W_{e} \equiv_{\mathrm{T}} K \Longrightarrow W_{f(e, 0)} \equiv_{\mathrm{T}} K$.

We now apply Theorem 4.4 with $W_{e}$ playing the role of $B, K$ starring as $C$, $W_{f(e, 0)}$ as $A_{0}$, and $W_{f(e, 1)}$ as $D$. By uniformity of the construction,

- $W_{e}<_{\mathrm{T}} K \Longrightarrow \begin{aligned} & \cdot W_{e}<_{\mathrm{T}} W_{f(e, 1)}<_{\mathrm{T}} K \text { and } \\ & \left.\cdot W_{f(e, 0)}\right|_{\mathrm{T}} W_{f(e, 1)} \text {, whereas }\end{aligned}$

- $W_{e} \equiv_{\mathrm{T}} K \Longrightarrow W_{f(e, 1)} \equiv_{\mathrm{T}} K$.

Iterating Theorem 4.4 with $A_{1}=W_{f(e, 1)}, A_{2}=W_{f(e, 2)}$ and so on yields the desired result in the case $n=0$.

For the case $n \geq 1$, the construction can be relativized, and then the corollary follows from $n$ applications of Theorem 4.5.

For a proof sketch of Theorem 4.2, we refer back to [31, Corollary 3.8], the weaker result that $\operatorname{MIN}_{\varphi}^{T^{(n)}}$ is Turing equivalent to $\emptyset^{(n+4)}$ for some Kolmogorov numbering $\varphi$. The lower bound of Theorem 4.2 is obtained by replacing "LOW" ${ }^{n}$ " in [31, Lemma 3.3(II)] with "HIGH ${ }^{n}$," and then using the corresponding function $f$ from Corollary 4.8, which deals with high sets, in place of the so-called function " $a$ " which deals with low ones. Combined with Theorem 4.7, this method gives $\operatorname{MIN}_{\psi}^{\mathrm{T}^{(n)}} \geq_{\mathrm{tt}} \emptyset^{(n+4)}$. The reverse bound follows from the fact that $\operatorname{MIN}_{\psi}^{\mathrm{T}^{(n)}} \in \Sigma_{n+4}$.

Acknowledgments. We would like to thank the anonymous referees for a very careful proofreading and numerous helpful comments. 


\section{References}

[1] Manuel Blum. On the size of machines. Information and Control, 11:257-265, 1967.

[2] Cristian S. Calude and André Nies. Chaitin $\Omega$ numbers and strong reducibilities. Journal of Universal Computer Science, 3(11):1162-1166 (electronic), 1997.

[3] John Case. Homework assignment for students. Computer and Information Sciences Department, University of Delaware, 1990.

[4] Gregory J. Chaitin. A theory of program size formally identical to information theory. Journal of the ACM, 22:329-340, 1975.

[5] Gregory J. Chaitin. Incompleteness theorems for random reals. Advances in Applied Mathematics, 8(2):119-146, 1987.

[6] George Davie. Foundations of mathematics - recursion theory question. http://cs.nyu.edu/pipermail/fom/2002-May/005535.html.

[7] Rodney G. Downey and Denis R. Hirschfeldt. Algorithmic randomness and complexity. Theory and Applications of Computability. Springer, New York, 2010.

[8] Stephen Fenner and Marcus Schaefer. Bounded immunity and bttreductions. Mathematical Logic Quarterly, 45(1):3-21, 1999.

[9] Santiago Figueira, Frank Stephan and Guohua Wu. Randomness and universal machines. Journal of Complexity, 22:738-751, 2006.

[10] Harvey Friedman. Foundations of mathematics - recursion theory question. http://cs.nyu.edu/pipermail/fom/2002-February/005289. html.

[11] Edward R. Griffor. Handbook of Computability Theory. Studies in Logic and Foundations of Mathematics. North Holland, Amsterdam, 1999.

[12] Sanjay Jain, Frank Stephan and Jason Teutsch. Index sets and universal numberings. CiE 2009: Proceedings of the 5th Conference on Computability in Europe, pages 270-279, Berlin, Heidelberg, 2009. SpringerVerlag. 
[13] Efim Kinber. On btt-degrees of sets of minimal numbers in Gödel numberings. Zeitschrift für Mathematische Logik und Grundlagen der Mathematik, 23(3):201-212, 1977.

[14] Martin Kummer. personal communication.

[15] Martin Kummer. On the complexity of random strings (extended abstract). STACS '96: Proceedings of the 13th Annual Symposium on Theoretical Aspects of Computer Science, pages 25-36, London, UK, 1996. Springer-Verlag.

[16] Martin Kummer and Frank Stephan. Recursion-theoretic properties of frequency computation and bounded queries. Information and Computation, 120(1):59-77, 1995.

[17] Per Martin-Löf. The definition of random sequences. Information and Control, 9:602-619, 1966.

[18] G. B. Marandžjan. On the sets of minimal indices of partial recursive functions. Mathematical Foundations of Computer Science, 74:372-374, 1979.

[19] G. B. Marandžjan. Selected topics in recursive function theory. Technical Report 1990-75, Technical University of Denmark, August 1990.

[20] Albert R. Meyer. Program size in restricted programming languages. Information and Control, 21:382-394, 1972.

[21] Piergiorgio Odifreddi. Classical recursion theory, volume 125 of Studies in Logic and the Foundations of Mathematics. North-Holland Publishing Co., Amsterdam, 1989. The theory of functions and sets of natural numbers, With a foreword by G. E. Sacks.

[22] Robert W. Robinson. Interpolation and embedding in the recursively enumerable degrees. Annals of Mathematics (2), 93:285-314, 1971.

[23] Gerald E. Sacks. Recursive enumerability and the jump operator. Transactions of the Amererican Mathematical Society, 108:223-239, 1963.

[24] Gerald E. Sacks. The recursively enumerable degrees are dense. Annals of Mathematics (2), 80:300-312, 1964.

[25] Marcus Schaefer. A guided tour of minimal indices and shortest descriptions. Archive for Mathematical Logic, 37(8):521-548, 1998. 
[26] Steven Schwarz. Quotient lattices, index sets, and recursive linear orderings. PhD thesis, University of Chicago, 1982.

[27] Robert I. Soare. Recursively enumerable sets and degrees. Perspectives in Mathematical Logic. Springer-Verlag, Berlin, 1987. A study of computable functions and computably generated sets.

[28] Frank Stephan. Degrees of computing and learning. Habilitationsschrift, University of Heidelberg, 1999.

[29] Frank Stephan. The complexity of the set of nonrandom numbers. In Cristian S. Calude, editor, Randomness and Complexity, from Leibnitz to Chaitin, pages 217-230. World Scientific, 2007.

[30] Frank Stephan and Jason Teutsch. Immunity and hyperimmunity for sets of minimal indices. Notre Dame Journal of Formal Logic, 49(2):107-125, 2008.

[31] Jason Teutsch. On the Turing degrees of minimal index sets. Annals of Pure Applied Logic, 148(1-3):63-80, 2007.

[32] Jason R. Teutsch. Noncomputable spectral sets. PhD thesis, Indiana University, 2007.

[33] A. K. Zvonkin and L. A. Levin. The complexity of finite objects and the development of the concepts of information and randomness by means of the theory of algorithms. Russian Mathematical Surveys, 25(6):83124,1970 . 